NOUVELLE

\section{Glioblastome multiforme}

\section{Les fleurs du MALT 1}

Clément Maghe ${ }^{1}$, Kathryn A. Jacobs ${ }^{1}$, Nicolas Bidère ${ }^{1}$, Julie Gavard ${ }^{1,2}$
l'Équipe SOAP (Signalisation en oncogenèse, angiogenèse et perméabilité),

CRCINA (centre de recherche en cancérologie et immunologie Nantes Angers), Inserm, CNRS, Université de Nantes, Université d'Angers, IRSUN (Institut de recherche en santé de l'université de Nantes), 8 quai Moncousu, 44000 Nantes, France. ${ }^{2}$ Institut de cancérologie de l'Ouest (ICO), Saint-Herblain, France. julie.gavard@inserm.fr
Le glioblastome multiforme, un cancer incurable

L'Organisation mondiale de la santé (OMS) a défini le glioblastome multiforme comme un cancer astrocytaire de grade IV. La survie médiane de cette tumeur cérébrale est d'environ 15 mois, malgré un traitement combinant chirurgie intracrânienne et séances concomitantes de radiothérapie et de chimiothérapie [1]. Les rechutes à proximité de la tumeur initiale, quasi inéluctables, évoluent rapidement et inexorablement jusqu'au décès du patient. Cet échec thérapeutique peut s'expliquer non seulement par les résistances intrinsèques des cellules tumorales, mais aussi par la présence d'un groupe minoritaire de cellules tumorales aux caractéristiques de cellules souches (glioblastoma stem-like cells, GSC). Les résultats de plusieurs travaux convergent vers l'idée que les GSC contrôlent l'initiation, la progression et la rechute de ces tumeurs [2], bien que les mécanismes en jeu restent mal compris. Ces cellules partagent de nombreuses caractéristiques avec les cellules souches neurales adultes, notamment la capacité d'autorenouvellement et de différenciation dans le lignage neural [2]. Éradiquer cette fraction de cellules tumorales apparaît donc comme une stratégie thérapeutique pertinente.

\section{MALT1 et la gliomagenèse}

Afin d'identifier des facteurs de vulnérabilité contrôlant l'expansion des GSC, les données transcriptomiques du TCGA (the cancer genome atlas) concernant plus de 400 patients atteints d'un glioblastome ont été interrogées. En se concentrant sur les gènes impliqués dans l'activation du facteur de transcription $N F-\kappa B$ (nuclear factor-kappa B), une voie essentielle dans la prolifération et la survie cellulaires dans de nombreux cancers $[3,4]$, la paracaspase MALTl (mucosa-associated lymphoid tissue lymphoma translocation protein 1) a émergé comme un nouvel acteur potentiel de la gliomagenèse [5]. L'expression de cette enzyme apparaît en effet corrélée à la fois au grade des tumeurs cérébrales et à la survie des patients atteints d'un glioblastome. Les fonctions cellulaires de MALTI sont doubles. D'une part, elle agit en tant que protéine adaptatrice au sein du complexe de protéines impliqué dans la mise en place de la voie de signalisation NF- $\mathrm{KB}[3,4]$, et, d'autre part, elle exerce une activité protéolytique à un site consensus de la séquence de ses protéines cibles, après un résidu arginine. Le nombre de substrats avérés de MALTl se limite à une dizaine, mais leur champ d'action s'étend du contrôle des voies NF-KB et JNK (jun N-terminal kinase), à l'adhérence cellulaire et à la stabilité des ARN [3] (Figure 1). La protéase MALTl, normalement maintenue dans une conformation inactive, est activée dans les cellules immunitaires pour contrôler la signalisation antigénique [3]. C'est aussi le cas dans les cellules non-immunitaires en réponse à l'activation de certains récepteurs membranaires (Figure 1). Pourtant, une étude biochimique du répertoire connu des substrats de MALTl montre qu'une fraction de cette protéase est constitutivement active dans les GSC
[5]. En accord avec ce résultat, l'expression d'une version de MALTl dépourvue d'activité catalytique permet de réduire la protéolyse de ses substrats dans les cellules de patients atteints d'un glioblastome. Nos résultats sont en faveur de l'idée que MALTl serait «piratée » dans le glioblastome, comme c'est le cas dans certains lymphomes [3-5].

\section{MALTl contribue à la survie des GSC} Par analogie avec les travaux démontrant l'addiction de cellules de lymphomes à l'activité de MALTI [3, 4], nous avons exploré l'impact de l'expression et de l'activité de cette protéase sur le maintien du réservoir de GSC et avons montré que la réduction de l'expression de MALTl dans des GSC induit une diminution de leur prolifération, de leur survie, et de l'expression de marqueurs de ces cellules [5]. Ces résultats ont été reproduits en utilisant des inhibiteurs de MALTl, tels que l'antagoniste peptidique compétitif z-VRPR-fmk, et des médicaments de la famille des phénothiazines, comme la molécule anti-psychotique mépazine, un inhibiteur allostérique de MALTl [4] (Figure 2). Ces agents pharmacologiques ont une toxicité sélective pour les GSC, et n'affectent pas la viabilité de cellules saines du cerveau (astrocytes, neurones, et cellules endothéliales) [5]. Finalement, l'administration journalière de mépazine permet de réduire de façon significative la croissance tumorale dans un modèle de souris greffées avec des GSC humaines [5]. Ces résultats montrent que la protéase MALTl est nécessaire au maintien des GSC et contribue à l'expansion tumorale. 


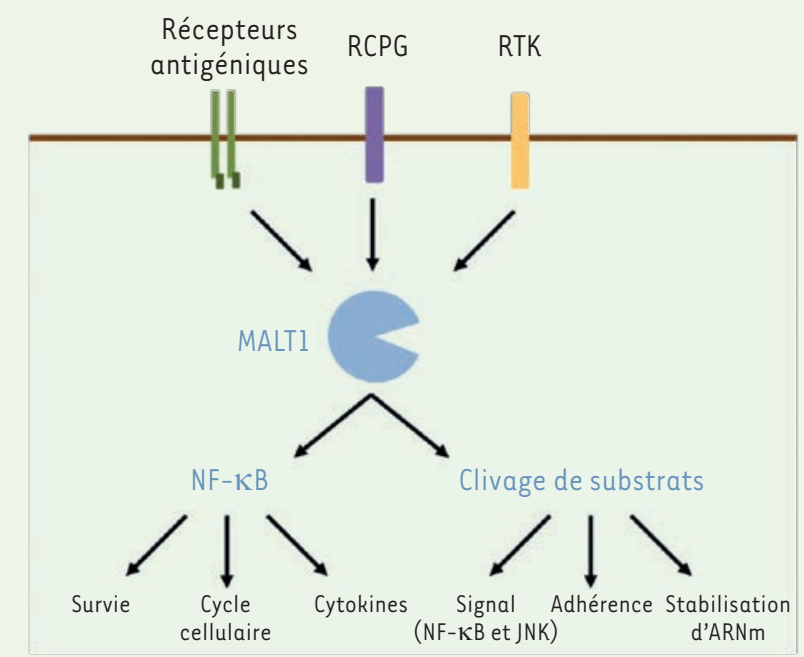

hibition de MALTl déconnecte mTOR des lysosomes et atténue la voie de signalisation mTOR tumorale [5], essentielle au maintien des GSC [10]. Ainsi, l'inactivation de MALTl entraîne une augmentation incontrôlée de la biosynthèse des lysosomes qui, en plus, semblent dysfonctionnels. En effet, nos résultats suggèrent que les lysosomes nouvellement formés ont une plus grande perméabilité de leur membrane, ce qui permet à des enzymes protéolytiques, normalement confinées à l'intérieur des lysosomes, d'exercer leur activité dans le cytosol. En outre, une analyse en imagerie confocale montre que les lysosomes nouvellement formés sont déconnectés de la voie de signalisation mTOR. Nos recherches s'attachent désormais à mieux caractériser les défauts de ces lysosomes.

Comment MALTI peut-elle moduler la quantité de lysosomes? L'action déterminante de quaking ( $(\mathrm{KI})$, une protéine qui se lie à l'ARN messager (ARNm), dans le maintien des GSC a récemment été rapportée [7]. Cette protéine permet en effet de stabiliser des ARNm codant des protéines endo-lysosomales. Nous avons montré que MALTl et QKI interagissent dans les GSC [5]. La surexpression de QKI, qui modifie la stœechiométrie du complexe MALTI/QKI, suffit à reproduire les effets de l'inhibition de MALTl dans les GSC. Inversement, la réduction de I'expression de QKI permet de contrer l'effet de l'inhibition de MALTl dans ces cellules [5]. Ainsi, il existe une interaction réciproque antagoniste entre ces deux protéines.

\section{Perspectives}

Nos travaux attribuent donc un nouveau rôle à la protéase MALTI dans I'homéostasie des lysosomes. Par la rétention de QKI, MALTl restreint la genèse du compartiment endo-lysosomal. Nous montrons également que l'activité de MALTl permet la signalisation mTOR, essentielle à l'expansion des GSC, renforçant l'idée que MALTI participe au contrôle de cette voie métabolique. En révélant 


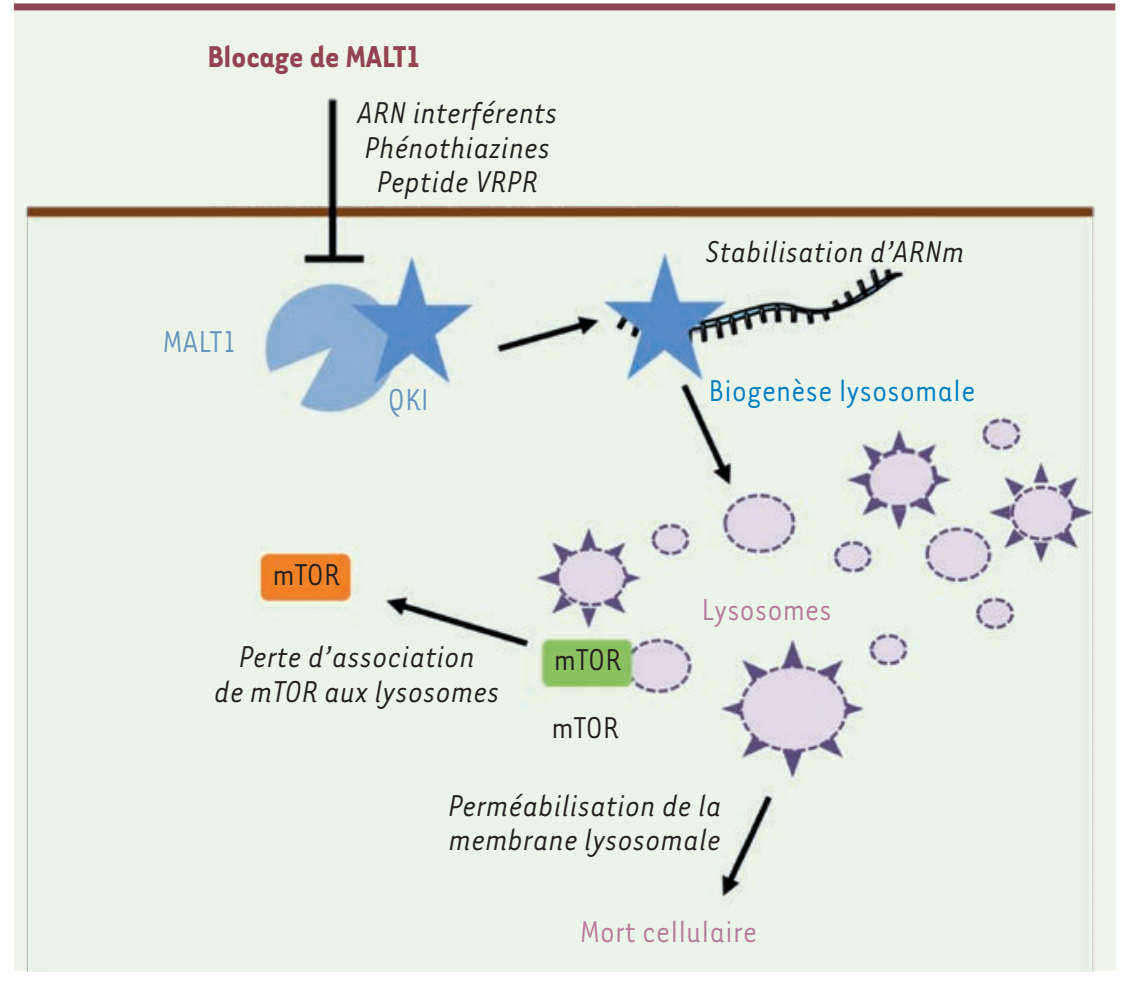

le rôle de la protéase MALTl dans le contrôle de la quantité et de l'activité des lysosomes qui, lorsqu'ils sont en excès, empêchent l'expansion des GSC, nos résultats ouvrent une perspective thérapeutique originale dans le combat contre le glioblastome multiforme. $\diamond$ MALT1 in glioblastoma:

the Flowers of Evil

\section{REMERCIEMENTS}

Nous tenons à remercier les membres présents et passés du laboratoire "Signalisation en oncogenèse, angiogenèse et perméabilité», Centre de recherche en cancérologie-immunologie de Nantes Angers, Inserm, CNRS, université de Nantes. Les projets de l'équipe sont soutenus par la fondation pour la recherche médicale, la fondation $A R C$, la Ligue contre le cancer, la Fondation de France, et l'Institut national du cancer.

\section{LIENS D'INTÉRÊT}

Les auteurs déclarent n'avoir aucun lien d'intérêt concernant les données publiées dans cet article.

\section{RÉFÉRENCES}

1. Stupp R, Mason WP, Van den Bent MJ, et al. Radiotherapy plus concomitant and adjuvant temozolomide for glioblastoma. $N$ Engl J Med 2005 ; $352:$ 987-96.

2. Lathia JD, Mack SC, Mulkearns-Hubert દ६, et al. Cancer stem cells in glioblastoma. Genes Dev 2015 ; 29: 1203-17.

3. Thys A, Douanne T, Bidere N. Post-translational modifications of the CARMAl-BCL10-MALTl complex in lymphocytes and activated B-cell-like subtype of diffuse large B-cell lymphoma. Front Oncol 2018 ; 8 : 498.

4. Nagel D, Spranger S, Vincendeau M, et al. Pharmacologic inhibition of MALTl protease by treatment of aggressive ABC-DLBCL. Cancer Cell 2012 ; $22: 825-37$.

5. Jacobs KA, André-Grégoire G, Maghe C, et al. Paracaspase MALTl regulates glioma cell survival phenothiazines as a therapeutic approach for the
Figure 2. MALTI est un agent essentiel de l'homéostasie des lysosomes dans les GSC. Dans les cellules de type «cellule souche » du glioblastome (GSC, glioblastoma stem-like cells), MALTI interagit avec la protéine quaking (QKI), une protéine de liaison à I'ARN. L'inhibition de l'activité enzymatique de MALTl par les phénothiazines ou par le peptide compétitif VRPR, ainsi que la réduction de son expression par des ARN interférents libère QKI, qui exerce alors son activité de protéine de liaison à I'ARN, et autorise la traduction d'ARN messagers (ARNm) codant des protéines du lysosome. II en résulte une abondance anormale des lysosomes. Les lysosomes nouvellement formés ont une membrane plus perméable aux protéases et sont déconnectés de la voie de signalisation mTOR. La perte d'intégrité de la membrane de ces lysosomes induit un déversement de leurs enzymes dégradatives dans le cytosol, conduisant à la mort des GSC.

by controlling endo-lysosome homeostasis. EMBO 2020 ; 39 : e102030.

6. Aits $S$, Jäättelä M. Lysosomal cell death at a glance. J Cell Sci 2013 ; 126: 1905-12

7. Shingu T, Ho A L, Yuan L, et al. Qki deficiency maintains stemness of glioma stem cells in suboptimal environment by downregulating endolysosomal degradation. Nat Genet 2017 ; 49 : 75-86.

8. Leeman DS, Hebestreit K, Ruetz T, et al. Lysosome activation clears aggregates and enhances quiescent neural stem cell activation during aging. Science 2018 ; 359 : 1277-83.

9. Le Joncour V, Filppu P, Hyvönen M, et al. Vulnerability of invasive glioblastoma cells to lysosomal membrane destabilization. EMBO Mol Med 2019; 11 : e9034.

10. Galan-Moya EM, Le Guelte A, Lima Fernandes $\varepsilon$, et al. Secreted factors from brain endothelial cells maintain glioblastoma stem-like cell expansion through the mTOR pathway. EMBO Rep $2011 ; 12$ : 470-6.

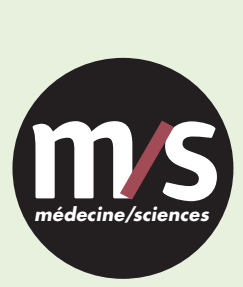

Tarifs d'abonnement $m / s-2020$

$>$ Grâce à $m / s$, vivez en direct les progrès des sciences biologiques et médicales

Abonnez-vous

à médecine/sciences

Bulletin d'abonnement page 538 dans ce numéro de $\mathrm{m} / \mathrm{s}$

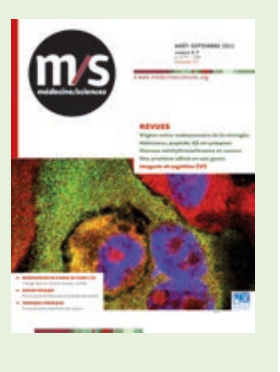

